\title{
Evolución de la cultura de seguridad del paciente en un hospital de media-larga estancia: indicadores de seguimiento
}

\section{Evolution of patient safety culture in a medium-stay hospital: monitoring indicators}

doi.org/10.23938/ASSN.0005

\author{
M.J. Merino Plaza' ${ }^{1-2}$, F.J. Carrera Hueso ${ }^{1}$, M. Castillo Blasco ${ }^{1}$, A. Martínez Asensi ${ }^{1}$, \\ R. Martínez Capella ${ }^{1}$, N. Fikri Benbrahim ${ }^{3}$
}

\section{RESUMEN}

Fundamento La seguridad del paciente es un reto para la asistencia sanitaria. El objetivo de este estudio es identificar las dimensiones con mayor impacto sobre la cultura de seguridad en un hospital de media-larga estancia, monitorizar su evolución y comprobar la sensibilidad de los indicadores de seguimiento.

Material y métodos Se realizaron 2 estudios transversales (2013-2015), utilizando la versión española del cuestionario "Hospital Survey on Patient Safety" (AHRQ). Variable resultado: alto grado de seguridad percibida (puntuación $\geq$ al percentil 75). Variables predIctoras: características socio-demográficas y percepciones de las dimensiones evaluadas. La asociación entre variables se cuantificó mediante OddsRatio.

Resultados El grado de seguridad percibida fue similar en ambos estudios: $\left(7,81-7,48 ; \mathrm{N}_{2013=66} ; \mathrm{N}_{201592}\right)$. Los aspectos mejor evaluados fueron: "Acciones de los supervisores" y "Trabajo en equipo en la unidad"; los peor evaluados fueron: "Dotación de personal", "Apoyo de la dirección" y "Trabajo en equipo entre unidades". Las variables socio-demográficas presentaron escasa significación, mientras que una percepción positiva de algunas dimensiones consideradas, sí que se asociaba a un alto grado de seguridad percibida. Los aspectos más fuertemente asociados fueron: "Acciones de los supervisores", "Franqueza en la comunicación" y "Problemas en los cambios de turno". En el estudio comparativo de los resultados, los indicadores basados en las dimensiones individuales detectaron mejor los cambios que el grado de seguridad percibida.

Conclusiones La evaluación periódica de la cultura de seguridad permite conocer el estado y la evolución de las percepciones de los profesionales. La elección de indicadores apropiados optimiza la información obtenida a través de estas encuestas.

Palabras clave. Cultura seguridad del paciente. Organizaciones sanitarias. Calidad asistencial.

\section{An. Sist. Sanit. Navar. 2017; 40 (1): 43-56}

1. Hospital Dr. Moliner.

2. Programa de Doctorado de Farmacia Social. Universidad de Granada.

3. Farmacia. Cátedra María José Faus de Atención Farmacéutica. Universidad de Granada.

Financiación: Este estudio ha sido realizado sin financiación externa.

Recepción: 12/11/2016

Aceptación provisional: 25/01/2017

Aceptación definitiva: 16/02/2017

\begin{abstract}
Background. Patient safety is a challenge for health care. The aim of this study is to identify the dimensions with the greatest impact on patient safety culture of healthcare staff in a medium-stay hospital; to asses its evolution over time and to check the sensitivity of monitoring indicators.
\end{abstract}

Methods. Two cross-sectional studies (2013 to 2015) were conducted. The safety culture assessment tool used was the Spanish version of the "Hospital Survey on Patient Safety" (AHRQ). Outcome variable is high perception of safety (score $\geq 75$ th percentile). Independent variables: socio-demographic characteristics and perceptions of the evaluated dimensions. The association between variables was quantified by Odds Ratio.

Results. The mean of perceived safety was similar in both studies: (7.81 and 7.48, $\mathrm{N}_{2013=66} ; \mathrm{N}_{2015=92}$ ). The best aspects evaluated were: "Supervisor actions" and "Teamwork within unit"; the worst evaluated were: "Staffing", "Management support" and "Teamwork across units". Socio-demographic variables had little significance, while a positive perception in some of the considered dimensions was associated with high perceived safety. The most strongly associated aspects were: "Supervisor actions", "Communication openness" and "Problems in transitions". In the comparative study, indicators based on individual dimensions detected changes better than the assessment of the degree of perceived safety.

Conclusion. The regular assessment of Patient Safety Culture makes it possible to know the status and evolution of professionals' perceptions. The choice of appropriate indicators optimises the information obtained through these surveys.

Keywords. Patient safety culture. Health care organizations. Healthcare quality.

\author{
Correspondencia: \\ María José Merino Plaza \\ Hospital Dr. Moliner \\ Carretera de PortaCoeli s/n \\ 46118-Serra (Valencia) \\ Correo electrónico: merino_mjo@gva.es
}

Conflicto de intereses: Los autores del presente artículo declaran no haber recibido financiación de ningún tipo y afirman que no existe conflicto de intereses laborales, de investigación, económicos ni morales para la realización del presente trabajo de investigación. 


\section{INTRODUCCIÓN}

La seguridad del paciente es un reto difícil de conseguir. Los eventos adversos (EA) forman parte de las preocupaciones diarias de los profesionales sanitarios ${ }^{1}$. Ya en los años 50, Barr $^{2}$ vio en ellos el precio a pagar por los modernos métodos diagnósticos y terapéuticos, mientras que Moser los denominó "las enfermedades del progreso de la medicina"3.Según la literatura, la mayoría de los EA son el resultado de la acumulación de errores humanos, fallos latentes del sistema y procesos organizacionales y administrativos. Diferentes estudios publicados desde los años 90 han contribuido al conocimiento de la magnitud, impacto y características de los EA ligados a la atención sanitaria ${ }^{4-8}$. El informe del año 1999 del Instituto Americano de Medicina To err is human ${ }^{9}$ hizo que muchos gobiernos situaran la seguridad del paciente entre sus prioridades de política sanitaria.

En España, la Estrategia de Seguridad del Paciente del Sistema Nacional de Salud (SNS) se desarrolló en 2005 y sus objetivos se orientaron a promover la cultura de seguridad, la gestión del riesgo, las prácticas seguras, la formación de los profesionales y la implicación de pacientes y ciudada$\operatorname{nos}^{10}$. A partir de estos objetivos se han desarrollado proyectos y programas para conocer mejor los problemas relacionados con la seguridad del paciente y las herramientas para prevenirlos.

Los programas ayudan a reducir significativamente los EA evitables, los costes asociados y la mortalidad hospitalaria ${ }^{11}$. Conseguir una adecuada cultura sobre la seguridad del paciente es la primera de las "buenas prácticas" recomendadas en el informe publicado el 2003 por el National Quality Forum ${ }^{12}$. Se ha sugerido que importantes palancas, como la formación o la mejora tecnológica, no producen los efectos esperados si no existe una cultura adecuada, capaz de sensibilizar a los profesionales sobre la importancia de este hecho. Por eso, es importante conseguir la concienciación e implicación del personal de enfermería, por su capacidad para liderar el cambio ${ }^{13}$.

Para comprobar la efectividad de las medidas adoptadas existen dos perspectivas. La primera se centra en establecer la magnitud del problema, promoviendo la declaración de los EA para disminuir su frecuencia a partir de su análisis y del diseño de barreras. La segunda incide en los elementos de la cultura y el clima de seguridad de la organización, los cuales determinan las prácticas de trabajo cotidianas y hacen que el personal perciba las diferentes dimensiones que influyen en la seguridad del paciente.

El mecanismo sugerido para abordar las dos perspectivas, es, por una parte, la evaluación permanente y proactiva de los EA producidos $\mathrm{y}$, por otra, monitorizar la evolución de la cultura de seguridad mediante cuestionarios validados ${ }^{13}$. Ésta es una potente herramienta de retroalimentación para generar interés entre el personal y para identificar las áreas prioritarias sobre las que intervenir. Las evaluaciones seriadas seguidas de intervenciones diseñadas en función de los resultados obtenidos, conducen a largo plazo a cambios en la cultura de seguridad de la organización ${ }^{14}$.

Existen pocos estudios sobre cultura de seguridad realizados en hospitales de media-larga estancia, en los que se atiende mayoritariamente a pacientes crónicos y de larga evolución, con gran dependencia y discapacidad, o en situaciones clínicas de enfermedad avanzada. En este tipo de centros, debido a la vulnerabilidad de sus pacientes, los EA relacionados con la atención sanitaria tienen una distribución diferente a la de los hospitales de agudos, tal como indican los resultados del estudio EARCAS $^{15}$. Este hecho planteó la necesidad de estudiar la cultura de seguridad del paciente de nuestros profesionales para analizar la situación y poder aplicar medidas correctivas en función de las necesidades detectadas.

Los objetivos de este trabajo fueron identificar las dimensiones con mayor im- 
pacto sobre la cultura de seguridad de los profesionales de un hospital de media-larga estancia, monitorizar su evolución y comprobar la sensibilidad de los indicadores de seguimiento empleados para evaluar los cambios.

\section{MATERIAL Y MÉTODOS}

Se llevó a cabo el estudio con el personal sanitario del Hospital Doctor Moliner.

Se realizaron dos estudios observacionales seriados (2013 y 2015) para evaluar la cultura de seguridad en un hospital público de media-larga estancia mediante un cuestionario validado. La participación del personal fue voluntaria y anónima. Antes de iniciar el estudio, se solicitó la aprobación de la Dirección y de las Comisiones de Calidad, Bioética y Docencia e Investigación del hospital.

El instrumento utilizado fue la versión española del cuestionario sobre seguridad del paciente Hospital Survey on Patient Safety (HSOPS) de la Agency for Health Care Research and Quality $(\mathrm{AHRQ})^{16}$. El cuestionario aplicado constaba de 3 partes. La primera incluía las preguntas que evaluaban 12 dimensiones vinculadas a la cultura de seguridad del paciente, mediante una escala Likert de 1 (menor grado de acuerdo con el enunciado) a 5 (acuerdo máximo). La segunda parte recogía una pregunta en la que el trabajador debía evaluar mediante una escala visual de cero a diez, el grado de seguridad percibida en la Unidad en la que prestaba sus servicios. La tercera parte recogía las características sociodemográficas y laborales de los encuestados. Al final del cuestionario, se incorporaba una pregunta abierta para incluir sugerencias. En el corte efectuado en 2013 no se evaluó la dimensión 1 relacionada con la notificación de EA, ya que todavía no se había implantado el sistema de notificación, pero en 2015 sí que se pasó el cuestionario completo.

El muestreo fue no probabilístico y consecutivo. En ambos momentos, la plantilla de personal sanitario del hospital era de 206 profesionales. En ambos estudios se procedió de la misma manera: la encuesta se entregó al personal a través del correo interno del hospital, junto a una carta en la que explicaban los objetivos del estudio y se solicitaba su colaboración, insistiendo en que la información recogida se utilizaría para buscar áreas de mejora. El cuestionario se distribuyó en febrero y las respuestas se recogieron durante el mes de marzo. El cuestionario fue auto-administrado y las respuestas se recogieron a través de los buzones de sugerencias para asegurar el anonimato.

Para describir las variables cuantitativas se utilizaron medidas de posición (media) y de dispersión (desviación estándar) y para las variables cualitativas o categóricas, se emplearon medidas de frecuencia (porcentajes). Para analizar las relaciones entre variables, se consideraron como variables independientes las características socio-demográficas de los trabajadores y sus percepciones sobre las diferentes dimensiones evaluadas. Como variable resultado se utilizó la alta percepción de seguridad, definida como una puntuación $\geq$ al percentil 75 del grado de seguridad percibida.

Para analizar la influencia de las distintas dimensiones sobre la alta percepción de seguridad, se codificó para cada una de ellas una nueva variable denominada "Evaluación Excelente de la dimensión", considerando que se alcanza este nivel cada vez que se respondía "5" (puntuación máxima) en todas las preguntas de la dimensión considerada ${ }^{17}$. Para el análisis bivariante se utilizó el test de Chi cuadrado para relacionar variables cualitativas, el test $t$ de Student para variables cuantitativas frente a variables cualitativas dicotómicas y el test de ANOVA para variables cuantitativas frente a variables cualitativas de 3 o más categorías.

Para estudiar la relación entre las dimensiones de la encuesta, se halló su matriz de correlación. Para cuantificar la asociación entre variables, se utilizó 
la Odds Ratio (OR) y su intervalo de confianza (IC) del 95\%. El análisis ajustado se realizó mediante regresión logística binaria, incluyendo en el modelo las variables potencialmente confundidoras. El modelo final se obtuvo por un método por pasos hacia delante y hacia detrás, al incorporar y retirar las variables consideradas que tuvieron una relación previa significativa con el resultado considerado.

El análisis estadístico se realizó con el programa IBM SPSS Statistics para Windows, versión 19.0.

Para la detección de puntos fuertes y áreas de mejora, se utilizaron los criterios de clasificación propuestos en el instrumento de evaluación ${ }^{16}$, considerándose fortalezas las dimensiones en las que más del 75\% de los participantes respondió positivamente (evaluación positiva, con puntuaciones de 4 o 5 ). Se consideraron puntos débiles las dimensiones en las que más del $50 \%$ de los participantes respondió negativamente (evaluación negativa de la dimensión, con puntuaciones de 1 o 2). Además, para mejorar la detección de los puntos débiles y facilitar la representación gráfica de los resultados, se aplicaron también los puntos de corte propuestos por Osakidetza-Servicio Vasco de Salud en su Manual de evaluación y mejora de la satisfacción de las personas en las organizaciones de servicios ${ }^{18}$, considerándose puntos débiles, las dimensiones con menos del $50 \%$ de respuestas positivas, puntos de mejora si el porcentaje de respuestas positivas era del $50-70 \%$, puntos fuertes si el porcentaje de respuestas positivas era del $70-80 \%$ y áreas excelentes si el porcentaje de respuestas positivas estaba por encima del $80 \%$.

\section{RESULTADOS}

El tamaño muestral no se predeterminó, ya que en los dos estudios se enviaron por correo interno los cuestionarios a todo el personal sanitario $(n=206)$ aunque en ambos momentos, solo devolvieron el acuse de recibo 158 profesionales; los 48 restantes no recibieron la encuesta por distintos motivos (baja laboral, libranza, días de libre disposición, etc). En 2013 se obtuvieron 66 respuestas (55 válidas), lo que representó una tasa de respuesta del $34,8 \%$. En 2015 se obtuvieron 92 respuestas (75 válidas), alcanzándose un índice de participación del 47,5\%. Algunos participantes no respondieron todas las variables socio-demográficas, pero sí contestaron las preguntas de la primera parte del cuestionario, considerándose la cumplimentación de la encuesta como válida.

La media del grado de seguridad percibida fue de $7,81(\mathrm{DE}=1,86)$ en 2013 y 7,48 $(\mathrm{DE}=1,99)$ en 2015 , sin diferencias estadísticamente significativas entre ambos momentos. El percentil 75 de la distribución en ambos casos fue de 9, por lo que las puntuaciones de 9 o 10, se catalogaron como "alta percepción de seguridad", frente al resto de posibles respuestas.

Los datos descriptivos de la plantilla y de los profesionales que respondieron a la encuesta en cada uno de los cortes realizados, se presentan en la tabla 1. El grado de seguridad percibida estratificado en función de las variables socio-demográficas consideradas (2013 y 2015), se presenta en la tabla 2. La edad media de los encuestados en 2013 fue de 43,70 años (DE=7,71) y en 2015 fue de 47,2 años (DE =8,2). En 2013 la muestra fue representativa en cuanto a la edad, categoría profesional y responsabilidad sobre dirección de equipos, observándose una mayor participación entre los hombres y el personal fijo. En 2015 la muestra fue representativa en cuanto a edad y categoría profesional, observándose mayor participación entre los hombres, el personal fijo, el personal con responsabilidad sobre dirección de equipos y entre los profesionales con contacto directo con el paciente. En el análisis bivariante, solo se obtuvieron diferencias estadísticamente significativas, en el corte realizado en 2013, para la variable "años de antigüedad en el hospital", con menor percepción de seguridad, en el grupo de entre 10 y 14 de antigüedad.

En 2013, las dimensiones más correlacionadas fueron: "Feed back en la comu- 
Tabla 1. Características de la población a estudio

\begin{tabular}{|c|c|c|c|c|}
\hline \multirow[b]{2}{*}{ Características sociodemográficas } & \multicolumn{2}{|c|}{ Año 2013} & \multicolumn{2}{|c|}{ Año 2015} \\
\hline & $\begin{array}{c}\text { Plantilla } \\
\text { personal } \\
\text { sanitario } \\
(n=206)\end{array}$ & $\begin{array}{c}\text { Respuestas } \\
\text { válidas } \\
(n=55)\end{array}$ & $\begin{array}{c}\text { Plantilla } \\
\text { personal } \\
\text { sanitario } \\
(n=206)\end{array}$ & $\begin{array}{l}\text { Respuestas válidas } \\
\qquad(\mathrm{n}=75)\end{array}$ \\
\hline Edad, media (DE) & $44,65(8,1)$ & $43,7(7,71)$ & $48,15(8,1)$ & $47,2(8,2)$ \\
\hline \multicolumn{5}{|l|}{ Sexo, n (\%) } \\
\hline Hombre & $29(14,1)$ & $8(21,1)$ & $28(13,5)$ & $14(19,7)$ \\
\hline Mujer & $178(85,9)$ & $30(78,9)$ & $178(86,5)$ & $57(80,3)$ \\
\hline \multicolumn{5}{|l|}{ Tipo de contrato, $\mathbf{n}(\%)$} \\
\hline Fijo & $51(24,8)$ & $17(32,7)$ & $53(25,6)$ & $27(37,5)$ \\
\hline No fijo & $155(75,2)$ & $35(67,3)$ & $153(74,4)$ & $45(62,5)$ \\
\hline \multicolumn{5}{|l|}{ Categoría profesional, n (\%) } \\
\hline Facultativos & $25(12,1)$ & $6(11,1)$ & $25(12,1)$ & $10(13,7)$ \\
\hline Enfermeros & $92(44,7)$ & $23(42,6)$ & $92(44,7)$ & $38(42,1)$ \\
\hline Auxiliar enfermería & $89(43,2)$ & $25(46,3)$ & $89(43,2)$ & $25(34,2)$ \\
\hline \multicolumn{5}{|l|}{ Dirección de equipos, n (\%) } \\
\hline No & $176(85,4)$ & $39(84,8)$ & $176(85,4)$ & $48(71,6)$ \\
\hline Sí & $30(14,6)$ & $7(15,2)$ & $30(14,6)$ & $19(28,4)$ \\
\hline \multicolumn{5}{|l|}{ Contacto directo con pacientes, $n$ (\%) } \\
\hline No & $30(14,6)$ & $8(14,8)$ & $30(14,6)$ & $5(6,8)$ \\
\hline Sí & $176(85,4)$ & $46(83,3)$ & $176(85,4)$ & $68(93,2)$ \\
\hline
\end{tabular}

nicación de errores" y "Franqueza en la comunicación" ( $\mathrm{r}=0,77)$, mientras que en 2015, la mayor correlación se observó entre "Respuesta no punitiva a los errores" y "Acciones de la supervisión que promueven la seguridad" $(r=0,66)$. La dimensión con mayor número de correlaciones $>0,50$ fue "Feed back en la comunicación de errores", con 4 coeficientes de correlación $>0,50$ en los dos cortes realizados.

Las OR ajustadas y su IC del 95\% se resumen en las tablas 3 y 4 . Al realizar el análisis estratificado por variables socio-demográficas se observó escasa significación, mientras que una evaluación excelente de algunas de las dimensiones consideradas, mostró asociación con el grado de seguridad percibida, especialmente tras realizar el análisis ajustado por regresión logística. Analizando la influencia de las variables socio-demográficas sobre el grado de seguridad percibida, únicamente mostraron significación el tipo de contrato, la categoría profesional y la antigüedad en el hospital, en el corte realizado en 2015 . El grado de seguridad percibida fue menor en el personal fijo, en los facultativos y en el personal con mayor antigüedad en el hospital.

Respecto al grado de asociación de las distintas dimensiones tras realizar el ajuste por posibles factores de confusión, en el estudio de 2015, los aspectos más fuertemente asociados al grado de seguridad percibida, con una $\mathrm{OR} \geq 10$, fueron: "Acciones de la supervisión que promueven la seguridad", "Franqueza en la comunicación" y "Problemas en los cambios de turno", siendo esta última dimensión la única que mostró asociación con el efecto en el estudio realizado en 2013 (Tabla 4). 
Tabla 2. Grado de seguridad percibida estratificado por las variables socio-demográficas de la población a estudio

\begin{tabular}{|c|c|c|c|c|c|}
\hline \multirow{2}{*}{\multicolumn{2}{|c|}{ Características socio-demográficas }} & \multicolumn{2}{|c|}{ Percepción seguridad 2013} & \multicolumn{2}{|c|}{ Percepción seguridad 2015} \\
\hline & & n (Porcentaje) & Media (IC 95\%) & n (Porcentaje) & Media (IC 95\%) \\
\hline \multirow[t]{5}{*}{ Edad } & $<35$ años & $5(9,8)$ & $8,2(6,6-9,8)$ & $7(9,6)$ & $8,2(7,0-9,4)$ \\
\hline & $35-44$ años & $24(47,1)$ & $7,9(7,2-8,6)$ & $11(15,1)$ & $6,8(5,2-8,4)$ \\
\hline & 45-49 años & $12(23,5)$ & $7,0(5,4-8,6)$ & $11(15,1)$ & $8,3(7,3-9,3)$ \\
\hline & 50-54 años & $5(9,8)$ & $8,1(5,2-11,0)$ & $14(19,2)$ & $7,3(6,1-8,4)$ \\
\hline & $\geq 55$ años & $5(9,8)$ & $7,9(6,6-9,2)$ & $30(41,1)$ & $7,4(6,6-8,1)$ \\
\hline \multirow[t]{2}{*}{ Sexo } & Hombre & $8(21,1)$ & $7,8(7,0-8,6)$ & $14(19,7)$ & $7,1(6,2-7,8)$ \\
\hline & Mujer & $30(78,9)$ & $7,6(6,9-8,3)$ & $57(80,3)$ & $7,5(7,0-8,1)$ \\
\hline \multirow[t]{5}{*}{ Tipo de contrato } & Fijo & $17(32,7)$ & $7,6(6,9-8,3)$ & $27(37,5)$ & $7,4(6,8-8,0)$ \\
\hline & Interino & $27(51,9)$ & $7,8(6,9-8,7)$ & $34(47,2)$ & $7,4(6,5-8,2)$ \\
\hline & Acúmulo de tareas & $1(1,9)$ & $10,0(10,0-10,0)$ & - & - \\
\hline & Eventual & $6(11,5)$ & $8,0(6,9-9,2)$ & $10(13,9)$ & $8,0(6,7-9,3)$ \\
\hline & Laboral & $1(1,9)$ & $7,0(7,0-7,0$ & $1(1,4)$ & $8,0(8,0-8,0)$ \\
\hline \multirow{3}{*}{$\begin{array}{l}\text { Categoría } \\
\text { profesional }\end{array}$} & Facultativos & $6(11,1)$ & $7,8(6,13-9,37)$ & $10(13,7)$ & $7,2(5,8-8,6)$ \\
\hline & Enfermeros & $23(42,6)$ & $8,1(7,7-8,6)$ & $38(42,1)$ & $7,2(6,6-7,9)$ \\
\hline & $\begin{array}{l}\text { Auxiliares } \\
\text { enfermería }\end{array}$ & $25(46,3)$ & $7,7(6,7-8,7)$ & $25(34,2)$ & $8,0(7,1-8,8)$ \\
\hline \multirow{5}{*}{$\begin{array}{l}\text { Antigüiedad en el } \\
\text { hospital }\end{array}$} & $<5$ años & $15(29,4)$ & $8,3^{*}(7,6-9,1)$ & $14(21,2)$ & $7,8(6,7-8,8)$ \\
\hline & De 5 a 9 años & $15(29,4)$ & $8,3(7,5-9,1)$ & $27(40,9)$ & $7,2(6,2-8,3)$ \\
\hline & De 10 a 14 años & $9(17,6)$ & $6,1^{*}(4,1-8,2)$ & $8(12,1)$ & $7,9(6,8-8,9)$ \\
\hline & De 15 a 19 años & $8(15,7)$ & $7,4(5,7-9,1)$ & $9(13,6)$ & $7,4(6,4-8,5)$ \\
\hline & $\geq 20$ años & $4(7,8)$ & $8,0(6,6-9,5)$ & $8(12,1)$ & $7,7(6,7-8,6)$ \\
\hline \multirow{2}{*}{$\begin{array}{l}\text { Dirección de } \\
\text { equipos }\end{array}$} & No & $39(84,8)$ & $7,8(7,1-8,4)$ & $48(71,6)$ & $7,7(7,0-8,2)$ \\
\hline & Sí & $7(15,2)$ & $8,2(7,4-9,0)$ & $19(28,4)$ & $7,4(6,7-8,2)$ \\
\hline \multirow{2}{*}{$\begin{array}{l}\text { Trabaja de } \\
\text { noches }\end{array}$} & No & $17(32,1)$ & $8,1(7,4-8,6)$ & $17(23,3)$ & $7,6(6,6-8,6)$ \\
\hline & Sí & $36(67,9)$ & $7,7(6,9-8,2)$ & $56(76,7)$ & $7,4(7,0-8,0)$ \\
\hline \multirow{2}{*}{$\begin{array}{l}\text { Trabaja los días } \\
\text { festivos }\end{array}$} & No & $9(19,6)$ & $8,5(7,9-9,1)$ & $18(24,7)$ & $7,5(6,5-8,4)$ \\
\hline & Sí & $37(80,4)$ & $7,6(7,0-8,2)$ & $55(75,3)$ & $7,5(6,9-8,0)$ \\
\hline \multirow{2}{*}{$\begin{array}{l}\text { Enfermedad } \\
\text { crónica }\end{array}$} & No & $43(81,1)$ & $7,9(7,2-8,4)$ & $54(74,0)$ & $7,4(6,8-7,9)$ \\
\hline & Sí & $10(18,9)$ & $7,5(6,4-8,5)$ & $19(26,0)$ & $7,8(7,1-8,5)$ \\
\hline \multirow{2}{*}{$\begin{array}{l}\text { Fármacos } \\
\text { ansioliticos }\end{array}$} & No & $47(87,0)$ & $7,7(7,2-8,3)$ & $58(80,6)$ & $7,6(7,0-8,1)$ \\
\hline & Sí & $7(13,0)$ & $8,3(7,0-9,0)$ & $14(19,4)$ & $6,9(5,8-8,0)$ \\
\hline \multirow{2}{*}{$\begin{array}{l}\text { Contacto directo } \\
\text { con pacientes }\end{array}$} & No & $8(14,8)$ & $8,4(7,6-8,8)$ & $5(6,8)$ & $7,7(6,0-9,0)$ \\
\hline & Sí & $46(85,2)$ & $7,7(7,0-8,2)$ & $68(93,2)$ & $7,5(7,0-7,9)$ \\
\hline
\end{tabular}

${ }^{*} \mathrm{p}<0,05$ 
Tabla 3. Asociación entre las variables socio-demográficas y el grado de seguridad percibida

\begin{tabular}{|c|c|c|c|c|c|}
\hline & & Grado de segurida & percibida 2013 & $\begin{array}{r}\text { Grado de seguri } \\
201\end{array}$ & $\begin{array}{l}\text { dad percibida } \\
5\end{array}$ \\
\hline Sociodemog & ráficas & $\begin{array}{l}\text { Alto grado de } \\
\text { seouridad nercibida }\end{array}$ & OR ajustada & $\begin{array}{l}\text { Alto grado de } \\
\text { seouridad }\end{array}$ & OR ajustada \\
\hline Edad & $<50$ años & $13 / 36(36,1)$ & $0,5(0,1-2,1)^{\mathrm{a}}$ & $15 / 46(32,6)$ & $4,4(0,6-30,7)^{\mathrm{a}}$ \\
\hline & $\geq 50$ años & $5 / 10(50,0)$ & 1 & $4 / 14(28,6)$ & 1 \\
\hline Sexo & Mujer & $11 / 30(36,7)$ & $1,5(1,2-12,2)^{\mathrm{b}}$ & $20 / 57(35,1)^{*}$ & No ajustable \\
\hline & Hombre & $3 / 8(37,5)$ & 1 & $0 / 14(0)$ & 1 \\
\hline Tipo de contrato & Fijo & $6 / 17(35,3)$ & $0,7(0,2-2,8)^{\mathrm{c}}$ & $6 / 27(22,2)$ & $\mathbf{0 , 2}(0,03-0,9)^{* \mathrm{c}}$ \\
\hline & No fijo & $15 / 35(42,9)$ & 1 & $16 / 45(35,6)$ & 1 \\
\hline Categoría & Facultativos & $2 / 6(33,3)$ & $0,4(0,04-5,5)^{\mathrm{d}}$ & $1 / 10(10)$ & $\mathbf{0 , 0 3}(0,001-0,8)^{\mathrm{d}^{*} \mathrm{c}}$ \\
\hline & Resto & $20 / 48(41,7)$ & 1 & $21 / 63(33,3)$ & 1 \\
\hline Antigüedad & $<15$ años & $7 / 15(46,7)$ & $\mathbf{7 , 1}(1,5-32,8)^{* \mathrm{e}}$ & $7 / 23(30,4)$ & $1,2(0,3-4,9)^{\mathrm{e}}$ \\
\hline & $\geq 15$ años & $14 / 36(38,9)$ & 1 & $15 / 50(30)$ & 1 \\
\hline Antigüedad en el & $<15$ años & $7 / 24(29,2)$ & $3,0(0,4-21,7)^{f}$ & $17 / 49(34,7)$ & $\mathbf{8 , 3}(1,1-64,5)^{* f}$ \\
\hline & $\geq 15$ años & $13 / 27(48,1)$ & 1 & $3 / 17(17,6)$ & 1 \\
\hline Antigüedad en el & $<15$ años & $12 / 29(41,4)$ & $2,1(0,3-17,6)^{\mathrm{g}}$ & $16 / 54(29,6)$ & $1,7(0,2-12,1)^{\mathrm{g}}$ \\
\hline & $\geq 15$ años & $9 / 23(39,1)$ & 1 & $3 / 8(37,5)$ & 1 \\
\hline Dirección de & Sí & $4 / 7(57,1)$ & $1,8(0,21-14,7)^{\mathrm{h}}$ & $5 / 19(26,3)$ & $2,4(0,4-13,5)^{\mathrm{h}}$ \\
\hline & No & $16 / 39(41,0)$ & 1 & $15 / 48(31,3)$ & 1 \\
\hline Trabaja de noches & No & $6 / 17(35,3)$ & $0,9(0,2-4,6)^{\mathrm{i}}$ & $6 / 17(35,3)$ & $2,5(0,5-11,6)^{i}$ \\
\hline & Sí & $15 / 36(41,7)$ & 1 & $16 / 56(28,6)$ & 1 \\
\hline Trabaja los días & No & $5 / 9(55,6)$ & $1,9(0,4-9,4)^{j}$ & $6 / 18(33,3)$ & $1,3(0,3-5,7)^{\mathrm{j}}$ \\
\hline & Sí & $15 / 37(40,5)$ & 1 & $16 / 55(29,1)$ & 1 \\
\hline Enfermedad & No & $17 / 43(39,5)$ & $0,4(0,1-1,8)^{\mathrm{k}}$ & $17 / 54(31,5)$ & $2,5(0,5-13,7)^{\mathrm{k}}$ \\
\hline & Sí & $5 / 10(50,0)$ & 1 & $5 / 19(26,3)$ & 1 \\
\hline Fármacos & No & $17 / 47(36,2)^{*}$ & $\mathbf{0 , 0 7}(0,006-0,83)^{* 1}$ & $19 / 58(32,8)$ & $3,6(0,6-26,8)^{1}$ \\
\hline ansionucos & Sí & $5 / 7(71,4)$ & 1 & $2 / 14(14,3)$ & 1 \\
\hline Contacto directo & No & $4 / 8(50,0)$ & $1,5(0,3-7,8)^{\mathrm{m}}$ & $2 / 5(40)$ & $2,6(0,2-29,9)^{\mathrm{m}}$ \\
\hline & Sí & $18 / 46(39,1)$ & 1 & $40 / 68(29,4)$ & 1 \\
\hline
\end{tabular}

OR: Odds Ratio; IC: Intervalo de confianza; ${ }^{*} \mathrm{p}<0,05$

ajustado para categoría profesional, contacto directo con pacientes, franqueza en la comunicación y trabajo en equipo en la unidad

${ }^{\mathrm{b}}$ Ajustado para edad, categoría profesional, trabajo en equipo en la unidad y franqueza en la comunicación

${ }^{c}$ Ajustado para categoría profesional, contacto directo con pacientes, acciones de la supervisión en pro de la seguridad y trabajo en equipo en la unidad

${ }^{\mathrm{d}}$ Ajustado para tipo de contrato, contacto directo con pacientes, trabajo en equipo en la unidad y trabajo en equipo entre unidades

e Ajustado para responsabilidad sobre dirección de equipos, enfermedad crónica, franqueza en la comunicación y trabajo en equipo entre unidades

${ }^{\mathrm{t}}$ Ajustado para contacto directo con pacientes, fármacos para dormir, aprendizaje organizacional y problemas al cambio de turno

${ }^{g}$ Ajustado para tipo de contrato, fármacos para dormir, trabajo en equipo en la unidad y problemas al cambio de turno

${ }^{\mathrm{h}}$ Ajustado para categoría profesional, contacto directo con pacientes, percepción de seguridad y trabajo en equipo en la unidad

${ }^{i}$ Ajustado para antigüedad en el hospital, tipo de contrato, trabajo en equipo en la unidad y problemas al cambio de turno

iAjustado para edad, categoría profesional, franqueza en la comunicación y problemas al cambio de turno

${ }^{\mathrm{k}}$ Ajustado para edad, contacto directo con pacientes, expectativas de la supervisión y franqueza en la comunicación

${ }^{1}$ Ajustado para antigüedad en el puesto, tipo de contrato, trabajo en equipo en la unidad y problemas al cambio de turno

${ }^{\mathrm{m}}$ Ajustado para edad, categoría profesional, franqueza en la comunicación y trabajo en equipo en la unidad 
Tabla 4. Asociación entre las dimensiones consideradas y el grado de seguridad percibida

\begin{tabular}{|c|c|c|c|c|c|}
\hline & \multirow[b]{2}{*}{ Dimensiones } & \multicolumn{2}{|c|}{$\begin{array}{c}\text { Grado de seguridad percibida } \\
2013 \\
\end{array}$} & \multicolumn{2}{|c|}{$\begin{array}{c}\text { Grado de seguridad percibida } \\
2015 \\
\end{array}$} \\
\hline & & $\begin{array}{l}\text { Alto grado de } \\
\text { seguridad percibida } \\
\text { /total. n (\%) }\end{array}$ & $\begin{array}{l}\text { OR ajustada } \\
\text { (IC del } 95 \% \text { ) }\end{array}$ & $\begin{array}{l}\text { Alto grado de } \\
\text { seguridad percibida } \\
\text { /total. n (\%) }\end{array}$ & $\begin{array}{l}\text { OR ajustada } \\
\text { (IC del } 95 \% \text { ) }\end{array}$ \\
\hline \multirow{2}{*}{$\begin{array}{l}\text { Frecuencia de } \\
\text { EA notificados }\end{array}$} & Evaluación excelente de la dimensión & & & $8 / 16(50)$ & $\mathbf{6 , 5}(1,3-33,3)^{\mathrm{a}}$ \\
\hline & Resto de profesionales & & & $14 / 54(25,9)$ & 1 \\
\hline \multirow{2}{*}{$\begin{array}{l}\text { Percepción } \\
\text { global de } \\
\text { seguridad }\end{array}$} & Evaluación excelente de la dimensión & $9 / 13(69,2)^{*}$ & $7,2(0,9-56,5)^{\mathrm{b}}$ & $15 / 31(48,4)^{*}$ & $\mathbf{6 , 6}(1,6-26,7)^{\star \mathrm{b}}$ \\
\hline & Resto de profesionales & $13 / 40(32,5)$ & 1 & $7 / 41(17,1)$ & 1 \\
\hline \multirow{2}{*}{$\begin{array}{l}\text { Acciones de la } \\
\text { supervisión }\end{array}$} & Evaluación excelente de la dimensión & $10 / 26(38,5)$ & $0,5(0,1-2,0)^{\mathrm{c}}$ & $7 / 8(87,5)^{* *}$ & $\mathbf{2 2 , 5}(1,5-344)^{* \text { c }}$ \\
\hline & Resto de profesionales & $11 / 27(40,7)$ & 1 & $15 / 64(23,4)$ & 1 \\
\hline \multirow{2}{*}{$\begin{array}{l}\text { Aprendizaje } \\
\text { organizacional }\end{array}$} & Evaluación excelente de la dimensión & $7 / 12(58,3)$ & $1,1(0,2-6,2)^{\mathrm{d}}$ & $9 / 14(64,3)^{*}$ & $\mathbf{8 , 9}(1,9-41)^{* \mathrm{~d}}$ \\
\hline & Resto de profesionales & $15 / 42(35,7)$ & 1 & $12 / 57(21,1)$ & 1 \\
\hline \multirow{2}{*}{$\begin{array}{l}\text { Trabajo en } \\
\text { equipo en la } \\
\text { Unidad }\end{array}$} & Evaluación excelente de la dimensión & $11 / 20(55)$ & $2,8(0,7-11,0)^{\mathrm{e}}$ & $9 / 17(52,9)^{*}$ & $\mathbf{6 , 3}(1,3-31,1)^{* \mathrm{e}}$ \\
\hline & Resto de profesionales & $11 / 34(32,4)$ & 1 & $13 / 56(23,2)$ & 1 \\
\hline \multirow{2}{*}{$\begin{array}{l}\text { Franqueza } \\
\text { en la } \\
\text { comunicación }\end{array}$} & Evaluación excelente de la dimensión & $11 / 23(47,8)$ & $0,3(0,04-2,5)^{\mathrm{f}}$ & $9 / 14(64,3)^{*}$ & $14,2(2,4-83,8)^{* \mathrm{f}}$ \\
\hline & Resto de profesionales & $11 / 31(35,5)$ & 1 & $13 / 59(22,0)$ & 1 \\
\hline \multirow{2}{*}{$\begin{array}{l}\text { Feed-back y } \\
\text { comunicación } \\
\text { sobre errores }\end{array}$} & Evaluación excelente de la dimensión & $12 / 23(52,2)$ & $4,8(0,7-34,2)^{g}$ & $7 / 15(46,7)$ & $\mathbf{5 , 7}(1,2-27,4)^{* g}$ \\
\hline & Resto de profesionales & $9 / 29(31,0)$ & 1 & $15 / 56(26,8)$ & 1 \\
\hline \multirow{2}{*}{$\begin{array}{l}\text { Respuesta no } \\
\text { punitiva a los } \\
\text { errores }\end{array}$} & Evaluación excelente de la dimensión & $5 / 9(55,6)$ & $0,9(0,1-6,6)^{\mathrm{h}}$ & $1 / 3(33,3)$ & $0,2(0,003-11,3)^{\mathrm{h}}$ \\
\hline & Resto de profesionales & $16 / 44(36,4)$ & 1 & $20 / 67(29,9)$ & 1 \\
\hline \multirow{2}{*}{$\begin{array}{l}\text { Dotación de } \\
\text { personal }\end{array}$} & Evaluación excelente de la dimensión & $3 / 4(75)^{*}$ & $3,3(0,2-51,5)^{\mathrm{i}}$ & $3 / 3(100)^{*}$ & No ajustable \\
\hline & Resto de profesionales & $18 / 49(36,7)$ & 1 & $19 / 70(27,1)$ & 1 \\
\hline \multirow{2}{*}{$\begin{array}{l}\text { Apoyo de la } \\
\text { Dirección }\end{array}$} & Evaluación excelente de la dimensión & $5 / 8(62,5)$ & $2,9(0,5-17,7)^{j}$ & $0(0)$ & No ajustable \\
\hline & Resto de profesionales & $16 / 44(36,4)$ & 1 & $18 / 58(31)$ & 1 \\
\hline \multirow{2}{*}{$\begin{array}{l}\text { Trabajo en } \\
\text { equipo entre } \\
\text { unidades }\end{array}$} & Evaluación excelente de la dimensión & $2 / 4(50,0)$ & $0,3(0,02-3,7)^{\mathrm{k}}$ & $4 / 12(33,3)$ & $0,3(0,04-2,6)^{\mathrm{k}}$ \\
\hline & Resto de profesionales & $20 / 50(40,0)$ & 1 & $17 / 59(28,8)$ & 1 \\
\hline \multirow{2}{*}{$\begin{array}{l}\text { Problemas en } \\
\text { cambios de } \\
\text { turno }\end{array}$} & Evaluación excelente de la dimensión & $10 / 16(62,5)^{*}$ & $\mathbf{8 , 2}(1,5-43,1)^{* 1}$ & $5 / 9(55,6)^{*}$ & $13,9(1,1-181)^{* 1}$ \\
\hline & Resto de profesionales & $12 / 38(31,6)$ & 1 & $17 / 64(26,6)$ & 1 \\
\hline
\end{tabular}

OR: Odds Ratio; IC: Intervalo de confianza; * $\mathrm{p}<0,05$

${ }^{a}$ Ajustado para categoría profesional, antigüedad en el hospital, respuesta no punitiva y dotación de personal

${ }^{\mathrm{b}}$ Ajustado para antigüedad en el hospital, responsabilidad sobre dirección de equipos, trabajo en equipo entre unidades y problemas al cambio de turno

${ }^{\mathrm{c}}$ Ajustado para categoría profesional, tipo de contrato, aprendizaje organizacional y trabajo en equipo en la unidad

d Ajustado para antigüedad en el hospital, fármacos para dormir, feed back comunicación de errores y problemas al cambio de turno

e Ajustado para categoría profesional, tipo de contrato, acciones de la supervisión en pro de la seguridad del paciente y respuesta no punitiva a los errores

${ }^{\mathrm{f}}$ Ajustado para categoría profesional, responsabilidad sobre dirección de equipos, feed back comunicación de errores y respuesta no punitiva a los errores

${ }^{g}$ Ajustado para categoría profesional, responsabilidad sobre dirección de equipos, franqueza en la comunicación y respuesta no punitiva a los errores

${ }^{\mathrm{h}}$ Ajustado para edad, antigüedad en el hospital, aprendizaje organizacional y feed back comunicación de errores

${ }^{i}$ Ajustado para tipo de contrato, categoría profesional, acciones de la supervisión en pro de la seguridad del paciente y aprendizaje organizacional

¡Ajustado para contacto directo con pacientes, fármacos para dormir, dotación de personal y franqueza en la comunicación

${ }^{\mathrm{k}}$ Ajustado para responsabilidad sobre dirección de equipos, contacto directo con pacientes, franqueza comunicación y problemas al cambio de turno

${ }^{1}$ Ajustado para antigüedad en el hospital, fármacos para dormir, trabajo en equipo entre unidades y respuesta no punitiva 


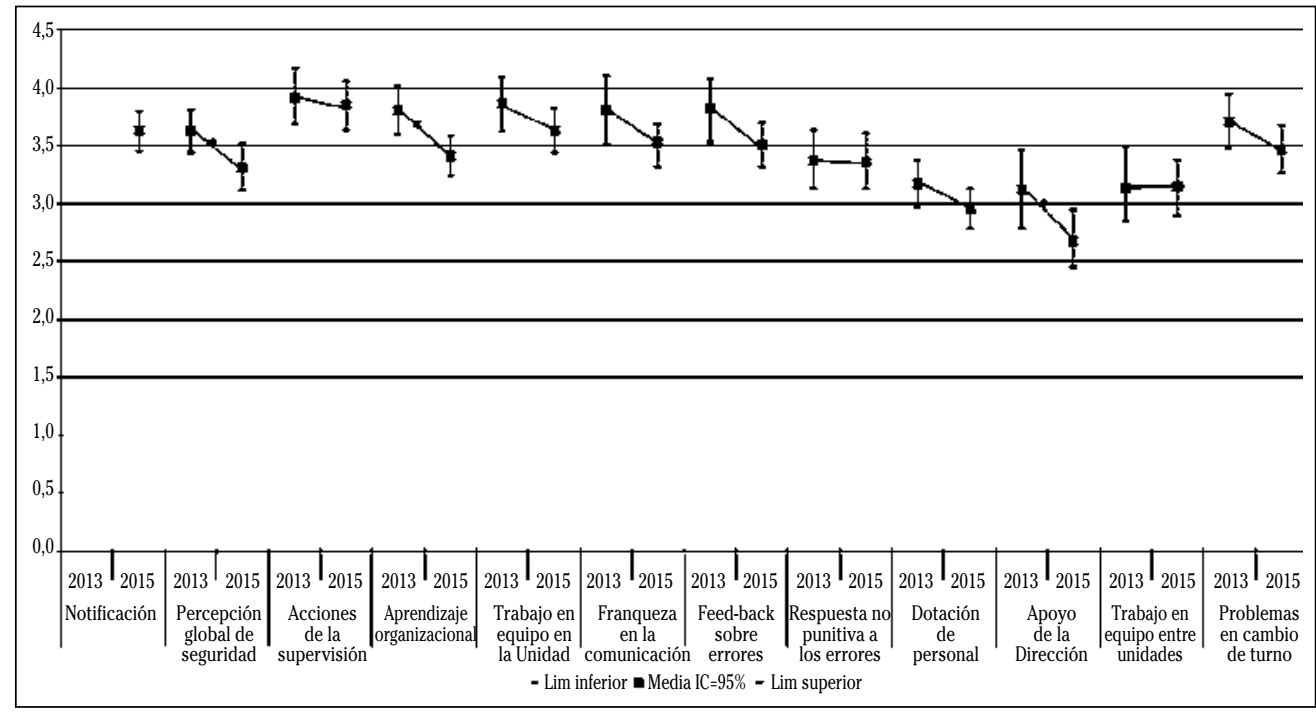

1: Notificación de eventos adversos; 2: Percepción global de seguridad; 3: Acciones de la supervisión que favorecen la seguridad del paciente; 4: Aprendizaje organizacional; 5: Trabajo en equipo en la Unidad; 6: Franqueza en la comunicación; 7: Feed-back y comunicación sobre errores; 8: Respuesta no punitiva a los errores; 9: Dotación de personal; 10: Apoyo de la Dirección a la seguridad del paciente; 11: Trabajo en equipo entre unidades; 12: Problemas en cambios de turno y transiciones entre servicios

Figura 1. Evolución de la seguridad media percibida respecto a cada una de las dimensiones consideradas (2013-2015)

En la figura 1 se representa la media de la seguridad percibida respecto a cada una de las dimensiones consideradas. Dicha media es similar en ambos momentos, observándose diferencias estadísticamente significativas únicamente para las dimensiones 2, 4 y 10 ("Percepción global de seguridad", "Aprendizaje organizacional" y "Apoyo de la dirección"), siendo menor la media obtenida en el corte realizado en 2015. Globalmente, los aspectos mejor evaluados en ambas encuestas fueron: "Acciones de la supervisión que promueven la seguridad" y "Trabajo en equipo en la unidad", mientras que los peor evaluados fueron: "Dotación de personal" y "Apoyo de la dirección". Siguiendo los criterios de clasificación propuestos por la $\mathrm{AHRQ}^{16}$, en nuestro estudio, no aparece como fortaleza ni como debilidad ninguna de las dimensiones en ninguno de los cortes realizados. Aplicando los puntos de corte propuestos por Osakidetza-Servicio Vasco de salud ${ }^{18}$, referidos al porcentaje de profesionales con percepción positiva de cada una de las dimensiones, sí que se detectan puntos fuertes y áreas de mejora, tal como se representa en la figura 2 . La representación gráfica de este indicador evidencia la existencia de áreas de mejora y pone de manifiesto las diferencias existentes entre los dos cortes realizados.

\section{DISCUSIÓN}

La tasa de respuesta fue inferior al 50\%, aunque en estudios similares realizados mediante cuestionarios auto-administrados y anónimos enviados al personal sanitario, es difícil superar esa tasa de participación ${ }^{19-22}$. Sin embargo, algunos autores obtuvieron índices de participación superiores al $70 \%$ en estudios realizados sobre colectivos más específicos o en el ámbito de atención primaria ${ }^{23,24}$. La tasa de participación pasó del 34,8\% en 2013 al 47,5\% en 2015 , lo que indica un cierto aumento en la adhesión al procedimiento. No obstante, aunque la muestra es representativa de la 
población total, el índice de participantes sigue siendo bajo.

Entre los distintos modelos de encuestas validadas considerados, se seleccionó el cuestionario HSOPS de la $\mathrm{AHRQ}^{16}$, porque cuenta con amplia experiencia en su aplicación y porque fue validado y adaptado al castellano por la Universidad de Murcia en 2005 en colaboración con el Ministerio de Sanidad. En el presente estudio, el grado de seguridad percibida fue adecuado, con una media de 7,81 y 7,48 respectivamente, resultado ligeramente superior al obtenido en otros estudios similares realizados en nuestro país. En el corte realizado en 2015, la seguridad percibida fue menor en el personal fijo, en los facultativos y en el personal con mayor antigüedad en el hospital. Estos resultados coinciden con los hallados en el estudio realizado por el Ministerio de Sanidad en $2008^{19,20}$, en el que la seguridad percibida por los médicos también fue inferior a la del resto de colectivos. Dicho estudio se realizó en 24 hospitales del SNS, sobre una muestra de 6.257 profesionales, a los que se aplicó el cuestionario HSOPS. El índice de participación fue del $40 \%$ y el grado de seguridad percibida fue de 7,0 puntos. En otro estudio realizado por SEMES en 2010 en 30 hospitales de 13 comunidades autónomas con la misma herramienta para evaluar la Cultura de seguridad en los Servicios de Urgencias Hospitalarias $^{21}$, el grado de seguridad percibida fue de 6,1. En otra evaluación realizada en 2012 en ocho hospitales españoles de la región de Murcia ${ }^{22}$, el índice de participación fue del $35,4 \%$ y el grado de seguridad percibida fue de 7,3.

Por contrapartida, en un estudio realizado en 2011 en el Servicio de Urgencias del Hospital Clínico Universitario de Zaragoza, en el que se aplicó el cuestionario HSOPS a médicos residentes ${ }^{24}$ la tasa de respuesta obtenida fue del $84,6 \%$ y el grado de seguridad percibida fue de 5,9. Es decir, al dirigirse el cuestionario a un colectivo más específico, la tasa de participación fue más alta que en los estudios dirigidos a colectivos más amplios, y al igual que otros estudios, el grado de seguridad percibido por los médicos, especialmente en los servicios de urgencias, fue inferior a los resultados obtenidos en otros estudios dirigidos a todo el personal sanitario.

Respecto a las dimensiones mejor y peor evaluadas por el personal, nuestros resultados son muy similares a los obtenidos en los estudios de referencia realizados en nuestro país. Los puntos de corte propuestos en la herramienta para la interpretación de resultados ${ }^{16}$ son poco sensibles y en la mayoría de estudios consultados, ninguna de las dimensiones estudiadas aparece como punto fuerte ni como área de mejora. Sin embargo, en casi todos ellos, destacan por el porcentaje de respuestas con carácter positivo dos de las dimensiones: "Acciones de la supervisión que promueven la seguridad", y "Trabajo en equipo en la Unidad". El porcentaje de respuestas positivas para estas dimensiones en el estudio realizado por el Ministerio en $2008^{19,20}$ fue del $61,8 \%$ y el $71,8 \%$ respectivamente, siendo estas dimensiones también las mejor evaluadas en el estudio realizado por SEMES $^{21}$ en 2010 y en otros estudios consultados $^{22-24}$. Ambas dimensiones tienen un carácter marcadamente interno al Servicio o Unidad en la que el profesional presta sus servicios, indicando que en general, se percibe que la mayoría de los problemas relacionados con la seguridad del paciente se originan o están relacionados con problemas externos al servicio o bien, con fallos de coordinación con otras unidades.

En relación a las debilidades u oportunidades de mejora percibidas por los encuestados, según los criterios de la $\mathrm{AHRQ}^{16}$, en nuestro estudio tampoco apareció ninguna, aunque si consideramos como áreas de mejora aquellas dimensiones en las que no se alcanza el $50 \%$ de respuestas positivas ${ }^{18}$, sí que hay 4 dimensiones que en alguno de los cortes realizados no alcanzaron esta puntuación (Fig. 2).

Estas dimensiones fueron: "Dotación de personal”, “ApoyodeDirección”,"Percepción global de seguridad" y "Trabajo en equipo entre Unidades". Estos resultados coinciden con los hallados en el estudio realizado en 2008 por el Ministerio ${ }^{19,20}$, con los del estudio realizado en 8 hospitales de la región de Murcia $^{22}$ y con el estudio realizado entre 


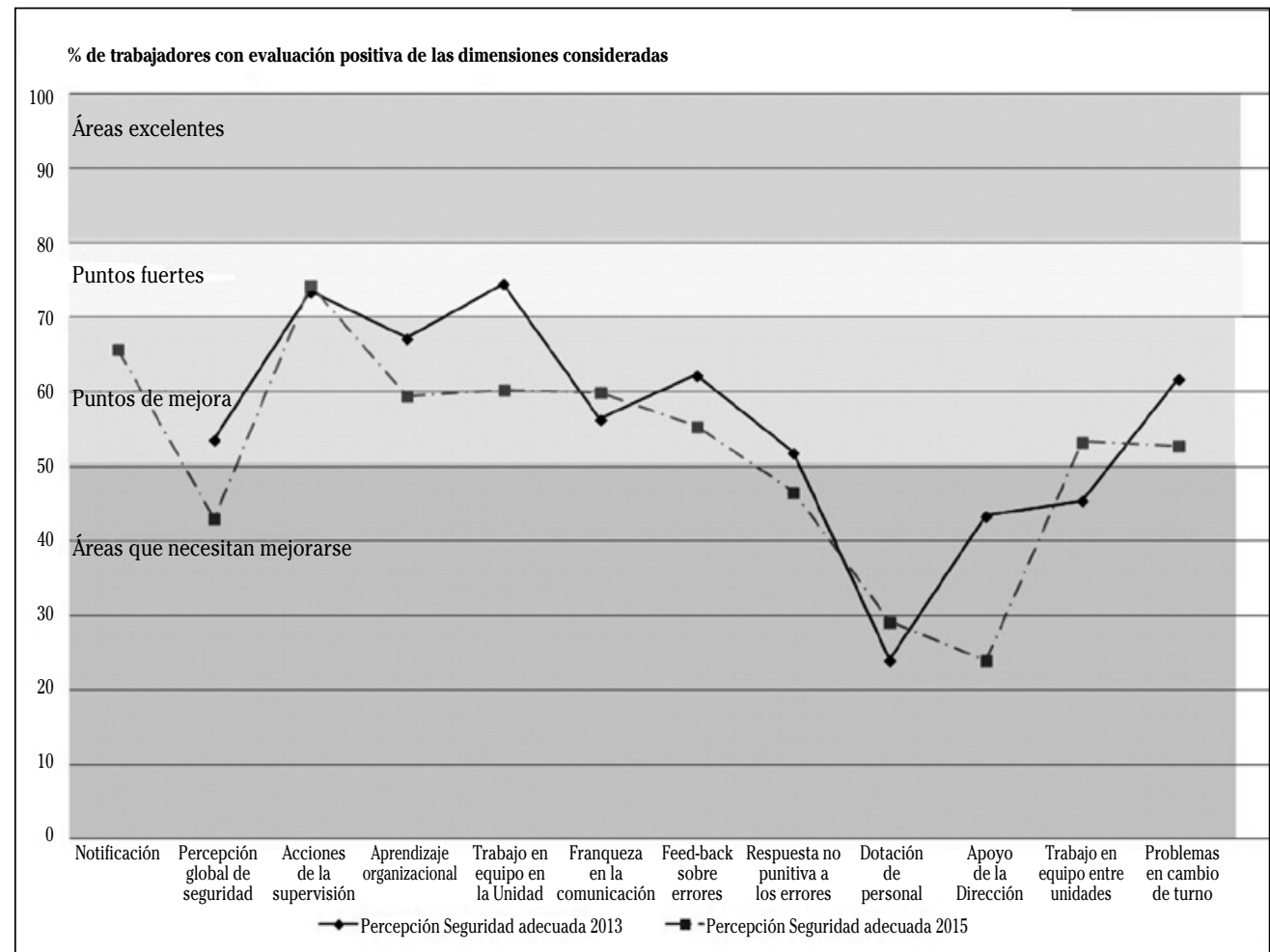

1: Notificación de eventos adversos; 2: Percepción global de seguridad; 3: Acciones de la supervisión que favorecen la seguridad del paciente; 4: Aprendizaje organizacional; 5: Trabajo en equipo en la Unidad; 6: Franqueza en la comunicación; 7: Feed-back y comunicación sobre errores; 8: Respuesta no punitiva a los errores; 9: Dotación de personal; 10: Apoyo de la Dirección a la seguridad del paciente; 11 : Trabajo en equipo entre unidades; 12: Problemas en cambios de turno y transiciones entre servicios

Figura 2. Porcentaje de trabajadores con evaluación positiva de las dimensiones consideradas (2013-2015)

médicos residentes en un servicio de urgencias hospitalario ${ }^{24}$. Resultados similares se obtuvieron en el estudio realizado por SEMES $^{21}$, en el que las dimensiones peor evaluadas también fueron la "Dotación de personal" y el "Apoyo de la Dirección", mientras que en un estudio realizado en un Área de Atención Primaria de Andalucía ${ }^{23}$, las dimensiones peor puntuadas fueron "Dotación de personal", "Respuesta no punitiva " y "Notificación de EA".

Son muchos los estudios que argumentan que la dotación de personal puede estar relacionada con los incidentes de seguridad, evidenciando la importancia de realizar una distribución racional del personal y una organización adecuada de los ritmos de trabajo a los que se ven someti- dos los profesionales, ya que este aspecto puede incrementar el riesgo de aparición de EA. Por otra parte, en todos los estudios consultados, el personal percibe que los supervisores tienen un mayor compromiso con la seguridad que los gestores, lo que pone de manifiesto la necesidad de mostrar una actitud más proactiva por parte de la gerencia en temas relacionados con la seguridad del paciente $\mathrm{e}^{19-25}$.

En general, la percepción de seguridad de 2015 fue inferior a la obtenida en 2013, aunque el grado de seguridad percibida se mantuvo en 7,5. Esta disminución pudo deberse al incremento del índice de participación y a la labor de concienciación, que hizo que el personal fuera más consciente de los posibles fallos del sistema. Estas 
variaciones en la percepción de seguridad han ido acompañadas de un importante incremento en la declaración de EA por parte del personal. En el periodo considerado se ha realizado seguimiento del número de EA declarados en el sistema de notificación SINEA, para evaluar la adhesión del personal al procedimiento. Tras la realización de diversas acciones de concienciación y la implantación del sistema de notificación a finales de 2013, se pasó de 8 EA declarados en 2013 , a 189 en 2014 y 299 durante el año 2015. Estos datos parecen indicar que la concienciación del personal ha aumentado y se declara más para tratar de aprender de los errores, hecho que parece confirmarse con la percepción del personal respecto a la dimensión 1 del cuestionario, que hace referencia a la notificación de EA, con una puntuación positiva del $66 \%$ de los participantes en la encuesta de 2015.

Según nuestros resultados, el grado de seguridad percibida se asoció con la evaluación excelente de ciertas dimensiones. El aspecto más fuertemente asociado en 2013 fue "Problemas en el cambio de turno", con una OR ajustada $\geq 5$. Los aspectos más fuertemente asociados con la percepción de seguridad en 2015 fueron "Acciones de la supervisión", "Franqueza en la comunicación" y "Problemas en los cambios de turno", con una OR ajustado $\geq 10$ (Tabla 4), siendo estas áreas aquellas en las que los planes de mejora podrían ser más efectivos en nuestra organización.

Respecto al seguimiento de la evolución de la cultura de seguridad, según nuestros resultados, la representación gráfica del porcentaje de trabajadores con evaluación positiva de seguridad respecto a cada una de las dimensiones consideradas, es el mejor indicador para monitorizar los cambios producidos. Dicho porcentaje aporta más información que la media de la seguridad percibida respecto a cada una de las dimensiones o que el grado de seguridad global percibida, indicadores que al menos, en nuestro caso, mostraron poca sensibilidad (Figs. 1 y 2).

Para ofrecer una atención sanitaria más segura, la comunicación efectiva entre los profesionales y con los pacientes es una herramienta clave de mejora ${ }^{26}$. El estilo de liderazgo, la personalidad y las actitudes de los directivos, pueden influir en las percepciones de los profesionales y en sus comportamientos y por tanto, tener importantes consecuencias para la seguridad del paciente ${ }^{25}$. La cultura organizacional es difícil de cambiar. Las personas pueden cambiar sus actitudes, pero este cambio es poco probable que sea sostenido sin un fuerte compromiso de la organización con la seguridad ${ }^{27}$. Todavía queda mucho por hacer para conseguir que el cambio de la cultura de las organizaciones sanitarias sea una realidad y que los directivos sean los impulsores de ese cambio, contando con la participación activa y real de todas las partes implicadas. Gran parte de las investigaciones en cultura de seguridad se han orientado a evaluar el clima de seguridad (actitudes y percepciones sobre seguridad que tienen los profesionales) y su asociación con los resultados clínicos, o con la satisfacción de profesionales y pacientes ${ }^{28}$. El impacto de las dimensiones que definen la cultura de seguridad, depende de las características propias de cada individuo y cada organización. La evaluación periódica de la cultura de seguridad es una herramienta útil para conocer el estado y la evolución de las percepciones de los profesionales y ayuda en la elaboración de planes de mejora. La periodicidad en la realización de estas encuestas debe ser suficiente para que las medidas adoptadas hayan tenido tiempo para su implantación efectiva y el colectivo haya percibido sus resultados. Sin embargo, el grado de seguridad percibido, puede ser un indicador poco sensible para el seguimiento de la eficacia de las acciones implementadas. En nuestro estudio, el grado de seguridad percibida es similar y adecuado en ambos cortes, pero al analizar las distintas dimensiones de forma individualizada, sí que se observan diferencias entre ambos estudios y se detectan áreas de mejora.

Entre las limitaciones de nuestro estudio, cabe destacar un posible sesgo de selección, ya que al ser la participación voluntaria y anónima, los profesionales más proactivos suelen ser también los más parti- 
cipativos. Otra limitación, es el bajo número de respuestas válidas, dado el pequeño tamaño de la organización y el incremento del índice de participación en el segundo corte realizado, lo que puede influir en la comparación de los resultados obtenidos. Por último, otro factor a considerar es la posible influencia de los cambios acaecidos durante el periodo considerado, con renovación del equipo directivo e intervenciones organizacionales que pudieron modificar las percepciones del personal.

En conclusión, los indicadores basados en la percepción individual de las dimensiones evaluadas; son más sensibles a los cambios que la evaluación del grado de seguridad percibida. Según nuestros resultados, obtenidos en un hospital de medialarga estancia, la representación gráfica del porcentaje de profesionales con una evaluación positiva de cada una de las dimensiones consideradas ha sido el indicador más sensible para detectar cambios.

\section{Agradecimientos}

A la Comisión de Calidad por los años de colaboración y esfuerzo para mejorar día a día la calidad asistencial.

\section{BIBLIOGRAFÍA}

1. Alberti KGMM. Medical errors: a common problem. BMJ 2001; 322: 501-502.

2. BARR DP. Hazards of modern diagnosis and therapy: the price we pay. J Am Med Assoc 1955; 159: 1452-1456.

3. Moser RH. Diseases of medical progress. N Engl J Med 1956; 255: 606-614.

4. Brennan TA, Leape LL, Laird NM, Hebert L, LoCALIO AR, LAWTHERS AG et al. Incidence of adverse events and negligence in hospitalized patients. Results of the Harvard Medical Practice Study I. N Engl J Med 1991; 324: 370376.

5. WiLSON RM, Runciman WB, GiBBERD RW, HARRISON BT, Newby L, Hamilton JD. The quality in Australian Health Care Study. Med J Aust 1995; 163: 458-471.

6. Nguyen TV, Hillman KM, Buist MD. Adverse events in British hospitals. BMJ 2001; $322: 1425$.
7. Estudio nacional sobre los efectos adversos ligados a la hospitalización: ENEAS 2005. Madrid: 2006. Ministerio de Sanidad y Consumo. [consultado 10-11-2016]: Disponible en: http://www.seguridaddelpaciente. es/resources/contenidos/castellano/2006/ ENEAS.pdf

8. Zegers M, De Bruijne MC, Wagner C, Hoonhout LHF, WaAiJman R, Smits M et al. Adverse events and potentially preventable deaths in Dutch hospitals: results of a retrospective patient record review study. Qual Saf Health Care 2009; 18: 297-302.

9. Institute of Medicine (US) Committee on Quality of Health Care in America. To Err is human: building a safer health system [Internet]. Kohn LT, Corrigan JM, Donaldson MS, editores. Washington (DC): National Academies Press (US); 2000 . [consultado 10-112016]: Disponible en: http://www.ncbi.nlm. nih.gov/books/NBK225182/

10 Estrategia seguridad del paciente 2015-2020. pdf. Ministerio de Sanidad;2015. [consultado 10-11-2016]: Disponible en: http://www.seguridaddelpaciente.es/resources/documentos/2015/Estrategia Seguridad del Paciente 2015-2020.pdf

11. Brilli RJ, McClead RE, Crandall WV, Stoverock L, Berry JC, WheELER TA et al. A comprehensive patient safety program can significantly reduce preventable harm, associated costs, and hospital mortality. J Pediatr 2013; 163 : 1638-1645.

12. The National Quality Forum: safe practices for better healthcare. A Consensus report. Washington: NQF; 2003.

13. Ginsburg L, Norton PG, Casebeer A, Lewis S. An educational intervention to enhance nurse leaders' perceptions of patient safety culture. Health Serv Res 2005; 40: 997-1020.

14. Chakravarty A, Sahu A, Biswas M, Chatterjee K, RATH S. A study of assessment of patient safety climate in tertiary care hospitals. Med J Armed Forces India 2015; 71:152-157.

15. Estudio EARCAS. Eventos Adversos en Residencias y Centros Asistenciales. Ministerio de Sanidad; 2011. [consultado 10-11-2016]: Disponible en: http://www.msssi.gob.es/organizacion/sns/planCalidadSNS/docs/EARCAS.pdf

16. Cuestionario sobre seguridad de los pacientes: versión española del Hospital Survey on Patient Safety. Madrid: Ministerio de Sanidad y Consumo; 2005. [consultado 10-11-2016]: Disponible en: http://www.msc.es/organizacion/sns/planCalidadSNS/docs/CuestionarioSeguridadPacientes1.pdf 
17. Robles-García M, Dierssen-Sotos T, MartínezOchoa E, Herrera-Carral P, Díaz-Mendi AR, LlORCA-DíAz J. Variables relacionadas con la satisfacción laboral: un estudio transversal a partir del modelo EFQM . Gac Sanit SESPAS 2005; 19: 127-134.

18. Servicio Vasco de Salud. Manual de evaluación y mejora de la satisfacción de las personas en las organizaciones de servicios. Vitoria-Gasteiz: Osakidetza Servicio Vasco de Salud; 2001. [consultado 10-11-2016]: Disponible en: http://www.osakidetza.euskadi. eus/contenidos/informacion/organizacion_ gestion_osk/es_og/adjuntos/satisfaccion/ evaluacion.pdf

19. Saturno PJ, da Silva-Gama ZA, de Oliveira-Sousa SL, Fonseca YA, de Souza-Oliveira AC, Castillo C et al. Análisis de la cultura de seguridad del paciente en los hospitales del Sistema Nacional de Salud español. Med Clin (Barc) 2008; 131: 18-25.

20. Analisis cultura SP en el ámbito hospitalario del Sistema Nacional de Salud Español. Ministerio de Sanidad; 2009. [consultado 1011-2016]: Disponible en: http://www.msc.es/ organizacion/sns/planCalidadSNS/docs/Analisis_cultura_SP_ambito_hospitalario.pdf

21. Roqueta-Egea F, Tomás-Vecina S, Chanovas-BoRRAS M. Cultura de seguridad del paciente en los servicios de urgencias: resultados de su evaluación en 30 hospitales del Sistema Na- cional de Salud español. Emergencias 2011; 23: 356-364.

22. da Silva Gama ZA, de Souza Oliveira AC, HernánDEZ PJS. Cultura de seguridad del paciente y factores asociados en una red de hospitales públicos españoles. Cad Saúde Pública 2013; 29: 283-293.

23. Pozo Muñoz F, Padilla Marín V. Evaluación de la cultura de seguridad del paciente en el ámbito de un área sanitaria. Rev Calid Asist 2013; 28: 329-336.

24. Jaraba Becerril C, Sartolo Romeo MT, Villaverde Royo MV, Espuis Albas L, Rivas Jiménez M. Evaluación de la cultura sobre seguridad del paciente entre médicos residentes de Medicina familiar y comunitaria en un servicio de urgencias hospitalario. An Sist Sanit Navar 2013; 36: 471-477.

25. Pronovost PJ, Weast B, Holzmueller CG, RosensTEIN BJ, KIDWELl RP, HALLER KB et al. Evaluation of the culture of safety: survey of clinicians and managers in an academic medical center. Qual Saf Health Care 2003; 12: 405-410.

26. Aranaz JM, Agra Y. La cultura de seguridad del paciente: del pasado al futuro en cuatro tiempos. Med Clínica 2010; 135: 1-2.

27. Firth-Cozens J. Evaluating the culture of safety. Qual Saf Health Care. 2003; 12: 401-401.

28. Flin R, Mearns K, O'Connor P, Bryden R. Measuring safety climate: Identifying the common features. Saf Sci 2000; 34: 177-192. 